\title{
Energy Efficiency Comparison between Co-located and Distributed Antenna Systems with D2D Communication
}

\author{
Xing-Quan $\mathrm{LI}^{1, \mathrm{a}}$, Chun-Long HE ${ }^{1}$, Ce ZHANG ${ }^{1}$ and Zi-Hao $\mathrm{LI}^{1}$ \\ ${ }^{1}$ Shenzhen Key Lab of Multi-dimensional Signal Processing, College of Information Engineering, Shenzhen \\ University, China
}

\begin{abstract}
In this paper, we investigate energy efficiency (EE) of co-located antenna system (CAS) and the distributed antenna system (DAS) with and without device-to-device (D2D) communications, respectively. We derive an approximate EE expression for both the idealistic and the realistic power consumption models in CAS and DAS with D2D communication. Numerical results show that the D2D communication is exactly benefited to improve the EE, especially in the DAS.
\end{abstract}

\section{Introduction}

Device-to-device (D2D) communication has been widely used to increase the cellular capacity, improve the energy efficiency (EE) of communication systems and extend the battery using time of UEs [1]-[3] because it can short the communication distances and reusing the channel resources with existing cellular users. It allows a user to communicate with another close user directly through D2D communication without extra communication through base stations. However, there are still many challenges when adding D2D links into the communication systems, which is mainly caused by the interference between cellular and the D2D users.

In fact, D2D underlaying cellular networks have attracted some research attention to improve the communication system performances [4]-[6]. Mode selection is an important part in D2D communication systems to further improve the performance of communication systems. In [7], the authors considered the mode selection problem for spectrum sharing between D2D links and cellular users in the cellular network. In [8], in order to prevent the excessive interference, the authors considered the transmission power control for D2D communications. To improve the scalability of D2D communications, authors of [9] discussed a distributed resource allocation method for D2D communications. Improving the reliability by interference management of D2D communications are considered in [10], [11].

Energy efficiency (EE) is becoming more and more important role for future communication systems due to the amount of energy consumption increasing dramatically with the exponential growth of service requirements [12]. In [13], three energy efficient modes switching scheme for single D2D pair has been considered to maximize energy efficiency under different conditions. For a multiinput multi-output (MIMO) architecture [14], [15] with multi-antenna base station is also an useful research area in design D2D communications underlaying such network models, which has not been

\footnotetext{
${ }^{a}$ Corresponding author: bedynamic@sina.com
} 
fully studied yet. What's more, the systems considered above mainly discussing contain D2D communications in the co-located antenna systems (CAS). However, to the best of the authors' knowledge, there is little investigation about D2D communications in the distributed antenna systems (DAS).

In this paper, we consider a cellular network with single UE and one D2D pair underlaying communications with base station antennas, which are located in different distributions, i.e., colocated antenna system (CAS) and distributed antenna system (DAS). We use the orthogonal frequency division multiple access (OFDMA) scheme [16] to study the effectiveness of the cellular energy efficiency by adding D2D communication into these two different models. Two different power consumptions models are considered. The first case only contains the overall transmit power. In the second case, beyond overall transmit power, we still consider the systems' dynamic power and static power consumptions. Numerical results show that the performance of DAS with D2D communication is much better than CAS.

The rest of this paper is organized as follows. Two different system models and two total power consumption models are presented, and the energy efficiency expression of the cellular are shown in section II. In Section III, numerical results are presented to demonstrated the effectiveness of DAS with D2D communication. In Section IV, concludes the paper and indications for future works directions are given.

\section{System models}

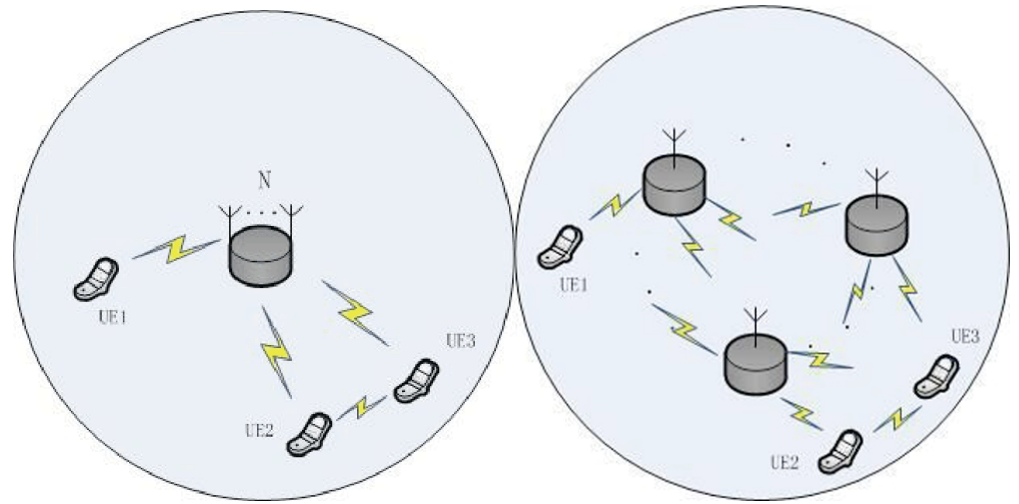

(a)

(b)

Figure 1. Two BS antenna layouts are considered in this paper: (a) With the CA system, all the BS antennas are located in the center of the cellular. (b) With the DA system, the BS antennas are uniformly distributed in the cellular.

We consider a downlink cellular network with three cellular user equipments (UEs), which are randomly distributed in the cellular. The cellular radius is $R . N$ base station (BS) antennas in the cellular are distributed in two different scenarios. The first scenario is all the base station antennas are co-located in the center of the cellular (CAS) and the other scenario is uniformly distributed in the cellular (DAS), which can be illustrated in the Figure 1. For simplicity, we assume that each UEs are equipped with single antenna and also assume that the total bandwidth is equally divided to each link between BSs and UEs so each UE is allocated with an orthogonal channel. We focus on the downlink performance of the $k$ th user. The received signal of user $k, k \in\{1,2,3\}$ can be represented as 


$$
y_{k}=\mathbf{h}_{k} \mathbf{x}_{k}+n_{k}
$$

where $n_{k} \sim \mathrm{CN}\left(0, N_{0}\right)$ is the additive white Gaussian noise (AWGN) at UE $k . \mathbf{x}_{k}$ is $N \times 1$ dimensional and $\mathbf{x}_{k} \sim \mathrm{CN}\left(0, P_{k}\right) . \mathbf{h}_{k}$ is $1 \times N$ dimensional vector, as the transmission signal vector and the channel gain vector from the BS to UE $k$, respectively. $\mathbf{h}_{k}$ denotes small scale and large scale fading channel, which can be modeled as

$$
\mathbf{h}_{k}=\gamma_{k} \circ \mathbf{g}_{k}
$$

where $\mathbf{g}_{k}$ denotes the $1 \times N$ small scale fading vector that can be modeled as independent and identically distributed complex Gaussian random variables with zero mean and unit variance and $\boldsymbol{\gamma}_{k}$ is the $1 \times N$ dimensional large scale fading vector [17]. For simplification, we ignore the shadowing effect and model the large scale fading coefficient as

$$
\gamma_{k, i}=\left\|p_{i}^{B}-p_{k}^{U}\right\|^{-\alpha / 2}
$$

where $p_{i}^{B}$ is the position of the $i$ th BS antenna and $p_{k}^{U}$ is the position of UE $k . \alpha$ is the pathloss factor and is typically between 3 and 5. Moreover, we assume that the channel state information (CSI) of all the involved links is perfectly known at BSs. The total transmission power of the BS is assumed to be fixed at $P_{t}$, and it is equally allocated to all the UEs, which is modeled as

$$
P_{i}=\frac{P_{t}}{3}, 1 \leq i \leq 3
$$

\section{Systems Analysis}

\subsection{Systems Rate Calculations}

In this paper, we normalize the total system bandwidth into unit. Orthogonal frequency division multiple access (OFDMA) is the access scheme for the downlink of the systems, i.e. the links between BS antennas and UEs use orthogonal channel to communicate with each other. The achievable rate of user $k$ can be represented as

$$
R_{k}=\frac{1}{3 N} \log _{2}\left(1 \frac{P_{k}\left\|\gamma_{k}\right\|^{2}}{N_{0}}\right)
$$

So the total downlink achievable rate of the cellular can be written as

$$
R_{\text {total }}=\sum_{k=1}^{3} R_{k}
$$




\subsection{Systems Rate Calculations with D2D}

We add D2D communication into the systems. Device-to-Device users are those who do not communicate through the BS antennas but communicate with each other directly. We assume that UE2 and UE3 are chosen as D2D user. We consider UE2 as D2D transmitter (Tx) has data to send to UE3 as D2D receiver $(\mathrm{Rx})$ and the distance between $\mathrm{Tx}$ and $\mathrm{Rx}$ is fixed as $L, L \ll R$, which D2D communication takes half of the bandwidth from the other UE and there is no interference between D2D and cellular communication. The achievable data rate at D2D Rx can be expressed as

$$
R_{D_{2 D_{-} k}}=\frac{1}{2 N} \log _{2}\left(1+\frac{P_{d} L^{-\alpha}}{N_{0}}\right)
$$

where $P_{d}$ is the transmitter power of Tx and $P_{d} \ll P_{k}$. So the data rate of UE1 can be expressed as

$$
R_{1}=\frac{1}{2 N} \log _{2}\left(1 \frac{P_{k}\left\|\gamma_{1}\right\|_{+}^{2}}{N_{0}}\right)
$$

It's easy to get the total achievable rate of the cellular with D2D communication, which can be written as

$$
R_{D 2 D_{-} \text {total }}=R_{1}+R_{D 2 D_{-} k}
$$

\subsection{Total Power Consumption Model}

As the related work discussed in [18], the realistic power consumption in the DAS contains three parts which can be written as

$$
P_{\text {real }}=\frac{P_{t}}{\tau}+L P_{d y}+P_{s t}
$$

where $\tau$ is the radio frequency power amplifier efficiency, $P_{d y}$ and $P_{s t}$ are the dynamic and the static power consumption, respectively. In the ideal condition, which means the circuit power is zeros. The idealistic power consumption can be expressed as

$$
P_{\text {ideal }}=\frac{P_{t}}{\tau}
$$

\subsection{EE Model}

As in [19], the EE model of the communication systems can be expressed as the ratio of the sum capacity and the total power consumption, which can be written as

$$
\eta_{E E}(R)=\frac{R}{P_{\text {Total }}}
$$


where $R$ is the sum capacity of the systems, which $R$ is equal to (6) when the systems without adding D2D communication, otherwise it can be expressed as (9). $P_{\text {total }}$ is equal to (10) when discussing the realistic power consumption model, otherwise $P_{\text {total }}$ is equal to (11).

\section{Simulation Results}

In this section, We present the different simulation results by comparing different scenarios, which are listed in the Table 1. Scenario 1 and 2 correspond to the traditional co-located antenna system model (CAS) and distributed antenna system model (DAS) without the D2D communications. Scenario 3 and 4 correspond to these different system models with green communications, i.e. with D2D communications. The system parameters in the simulations are listed in the Table 2. We average over 1000 randomly generated UEs locations and present the average results.

Table 1. Different investigated scenarios.

\begin{tabular}{c|cc}
\hline & System Model & D2D communication \\
\hline Scenario 1 & CAS & NO \\
Scenario 2 & DAS & NO \\
Scenario 3 & CAS & YES \\
Scenario 4 & DAS & YES \\
\hline
\end{tabular}

Table 2. Simulation parameters.

\begin{tabular}{c|c}
\hline Parameters & Value \\
\hline The cellular radius R & $1000 \mathrm{~m}$ \\
The D2D distance L & $100 \mathrm{~m}$ \\
The BS antenna number N & 5 \\
The noise power N0 & $-104 \mathrm{dBm}$ \\
The dynamic power consumption Pdy & $30 \mathrm{dBm}$ \\
The static power consumption Pst & $40 \mathrm{dBm}$ \\
The maximum transmit power Pt & $5 \sim 46 \mathrm{dBm}$ \\
Path loss exponent $\alpha$ & 4 \\
Drain efficiency $\boldsymbol{\tau}$ & $38 \%$ \\
\hline
\end{tabular}

From Figure 2., we show the total achievable rate of the overall cellular network with the four scenarios listed in the Table 2. It is obviously shows that the total achievable rate of DAS is much better than CAS under different maximum transmit power. After adding D2D communications, the total achievable rates of these two systems are apparently better than the original systems, which means D2D communication is a better way to increase the systems' total achievable rate.

From Figure 3., we present the results of EE of CAS and DAS in the ideal power consumption, with and without D2D communications under different maximum transmit power, respectively. We can easily find that in the condition of lower maximum transmit power, after adding D2D 
communications in the systems, since they can communicate with each other directly and save the energy consumption to communicate through BSs, EE of the systems will slightly increase. What's more, from the general view, it's easy to find that the systems EE decreases with the growth of the maximum transmit power in the idealistic power consumption model. And EE in the realistic power consumption model of these two systems under the same condition with Figure 3. are presented in the Fig 4., which apparently shows EE first increases and then decreases with the growth of the maximum transmit power. We can find the optimal transmit power to maximize EE is about $33 \mathrm{dBm}$. The results of these two figure is consistent with the theorems concluded in [20].

From Figure 5., we demonstrate the influence of systems EE when the D2D communications distance is changing under the maximum transmit power is set as $46 \mathrm{dBm}$. It's apparently that EE decreases with the growth of the D2D communication distance. The reason is, when the D2D communications distance growth, the achievable rate of D2D communications will slightly decrease, which causes the total achievable rate decreasing.

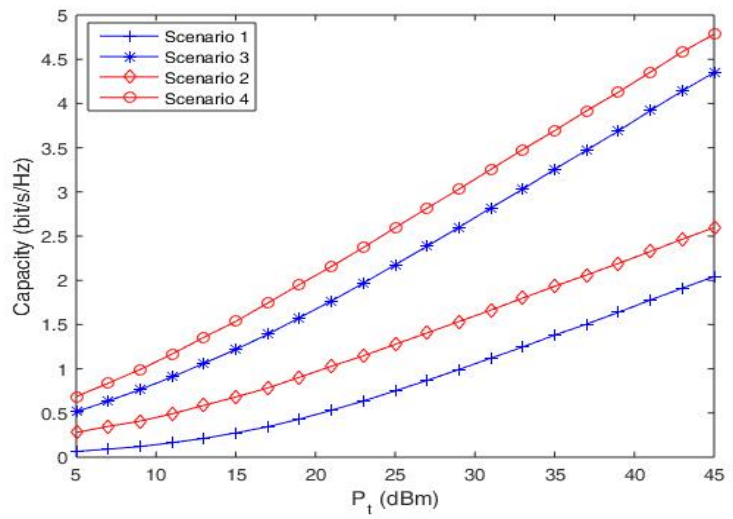

Figure 2. Capacity versus the maximum transmit power.

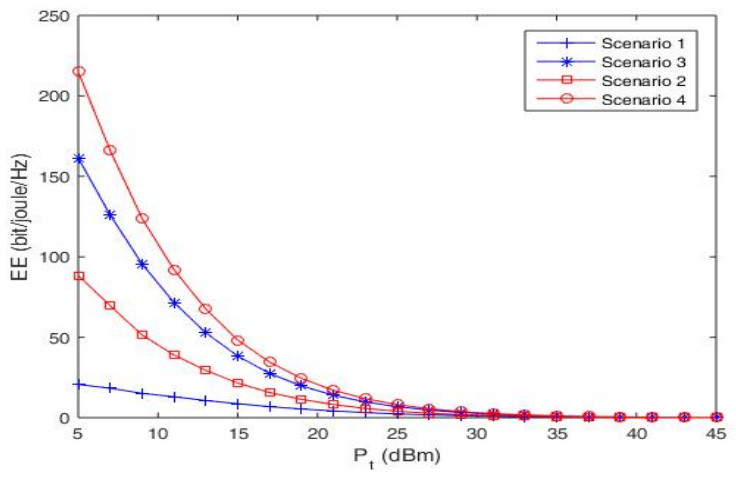

Figure 3. Energy efficiency in the ideal condition versus the maximum transmit power. 


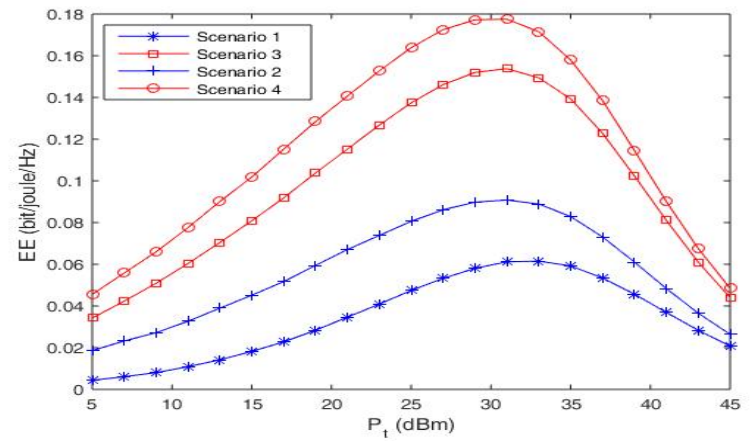

Figure 4. Energy efficiency in the real condition versus the maximum transmit power.

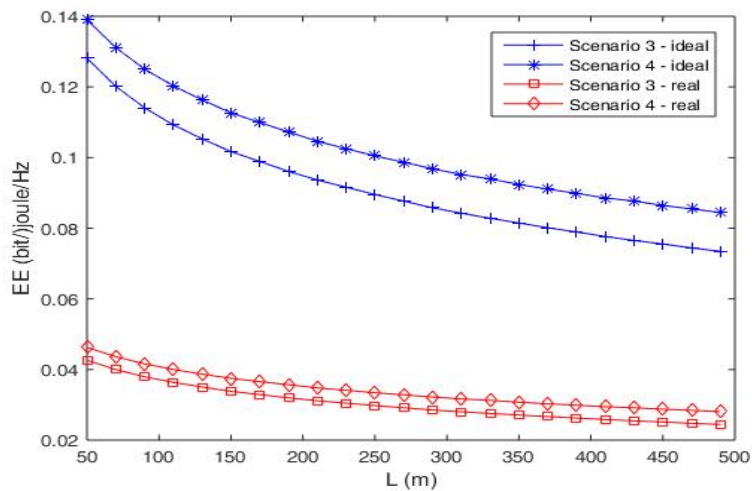

Figure 5. Energy efficiency in the ideal and real conditions versus the different D2D distances.

\section{Conclusion}

In this paper, we investigated the EE of the CAS and DAS, with and without D2D communications, respectively. We also find that when the systems adding D2D communications, the systems' total achievable rate remarkably increased and the same as the systems' EE, which is depended on the distance of D2D users. Simulation results also confirm it's correctness. What's more, adding D2D communications to the CAS and DAS is very useful to reduce energy efficiency consumption. Since all the scenarios only contain one D2D pair and a cellular user in a single cellular, we will extend it to multi-cells with more D2D pairs and cellular users in the future works.

\section{Acknowledgment}

This work is partially supported by the Natural Science Foundation of China under grants 61601300 , the Science and Technology Innovation Commission of Shenzhen under Grants JCYJ20150324140036835, the Open Research Fund through the National Mobile Communications Research Laboratory, Southeast University, Nanjing, China, under Grant 2017D10, Shenzhen University start-up funding No. 2016053. 


\section{References}

1. K. Doppler, M. Rinne, C. Wijting, IEEE Commun. Mag., 47, 12(2009)

2. G. Fodor, E. Dahlman, G. Mildh, IEEE Commun. Mag., 50, 3(2012)

3. C. H. Yu, K. Doppler, C. B. Ribeiro, IEEE Trans. Wireless Commun., 10, 8(2011)

4. Z. Zhou, M. Dong, K. Ota, IEEE Commun. Lett., 3, 5(2014)

5. F. W. F, C. X. C, L. Song, Proc. WCSP, 2012

6. T. D. Hoang, B. L, T. Le-Ngoc, Proc. IEEE ICC, 2015

7. M. Zulhasnine, C. Huang, and A. Srinivasan, Proc. IEEE WiMob'2010, 2010

8. C. H. Yu, O. Tirkkonen, K. Doppler, Proc. IEEE VTC, 2009

9. N. Reider, G. Fodor, EURASIP Journal on Wireless Commun.and Networking, 2012, 1(2012)

10. H. Min, W. Seo, J. Lee, IEEE Trans. Wireless Commun., 10, 2(2011)

11. Y. Xu, R. Yin, T. Han, Proc. IEEE ICCC, 2012

12. G. Fettweis, E. Zimmermann, Proc. WPM Commun., 4(2008)

13. D. Feng, G. Yu, C. Xiong, IEEE Trans. Wireless Commun., 14, 12(2015)

14. Q. H. Spencer, C. Peel, A. L. Swindlehurst, IEEE Commun. Mag., 42, 10(2004)

15. D. Gesbert, M. Kountouris, R. W. H. Jr., C. Chae, T. Salzer, IEEE Signal Proc. Mag., 4, 5(2007)

16. C. He, G. Y. Li, F Zheng, IEEE Trans. Vehicular Technology, 63, 3(2014)

17. C. He, B. Sheng, P. Zhu, IEEE J. Sel. Areas Commun., 31, 5(2013)

18. D. W. K. Ng, E. S. Lo, IEEE Trans. Wireless Commun., 11, 9(2012)

19. Y. Rui, Q. T. Zhang, L. Deng, IEEE J. Sel. Areas Commun., 31,5(2013)

20. C. L. He, J. Yin, Y. He, Journal of Commun. and Networks, 18, 4, (2016) 